$\begin{array}{cc}\text { ACADEMIA ROMÂNĂ } & \text { Rev. Roum. Chim., } \\ \text { Reve, 65(6), 567-572 } \\ \text { Rttp://web.icf.ro/rrch/ } & \text { DOI: } 10.33224 / \text { rrch.2020.65.6.06 } \\ & \end{array}$

\title{
CLICK SYNTHESIS AND COMPLEXATION PROPERTIES OF A NEW UNSYMMETRICAL MACROCYCLE BEARING 1,4-DIOXABENZENE AND TRIAZOLE UNITS ${ }^{* *}$
}

\author{
Teodor A. CUCUIET, a Cătălin C. ANGHEL,,${ }^{\mathrm{a}, \mathrm{b}}$ Elena BOGDAN, ${ }^{\mathrm{a}}$ Andreea CRIŞAN, a \\ Mihaela MATACHE, ${ }^{\mathrm{b}}$ Lidia POP, ${ }^{\mathrm{a}}$ Anamaria TEREC ${ }^{\mathrm{a}}$ and Niculina D. HĂDADE ${ }^{\mathrm{a},{ }^{,}}$ \\ ${ }^{a}$ Babeș-Bolyai University, Faculty of Chemistry and Chemical Engineering, Supramolecular Organic \\ and Organometallic Chemistry Centre, 11 Arany Janos Str., RO-400028-Cluj-Napoca, Roumania \\ ${ }^{b}$ University of Bucharest, Faculty of Chemistry; Department of Organic Chemistry, Biochemistry and Catalysis, \\ Research Centre of Applied Organic Chemistry, 90-92 Panduri Street, RO-050663 Bucharest, Roumania
}

We report herein synthesis of a new triazole-based unsymmetrical macrocycle through Copper(I) catalyzed Alkyne - Azide Cycloadditon (CuAAC), using a copper(I)- $N$-heterocyclic carbene complex as catalyst. The obtained macrocycle was characterized by NMR spectroscopy and High Resolution Mass Spectrometry (HRMS). The complexation ability of the macrocycle towards various cations as well as its selectivity for a particular metal-ion was investigated by HRMS experiments.

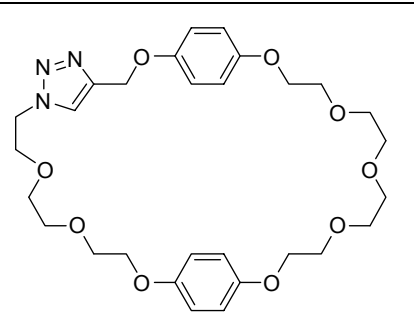

\section{INTRODUCTION}

Macrocycles and their host-guest complexes hold a central place in supramolecular chemistry. ${ }^{1}$ To date, there are several well established procedures that enable efficient preparation of macrocyclic compounds such as high dilution technique, use of ions or small molecules as templates and dynamic combinatorial chemistry as well as combination of these techniques. ${ }^{1,2}$ One of the key requirements for the preparation of this type of compounds is use of a highly efficient reaction in the macrocyclization step. ${ }^{3}$

Copper(I) catalyzed alkyne - azide cycloadditon (CuAAC) is one of the most widely used click reaction that found applications in many fields ranging from supramolecular chemistry ${ }^{4}$ to material sciences ${ }^{5}$ and medicinal chemistry. ${ }^{6}$ One of the most preeminent applications in supramolecular chemistry is the synthesis of mechanically interlocked structures such us rotaxanes $^{7}$ and catenanes ${ }^{8}$ mainly due to the orthogonality of the reactive groups, mild reaction conditions (compatible with the noncovalent interactions required for building up these molecules) and its high yields.

This reaction has also been applied for synthesis of macrocycles with ion recognition properties. ${ }^{9}$ Relevant examples include macrocyclic peptides, ${ }^{10}$ sugar-containing macrocycles, ${ }^{11}$ crown ether ${ }^{12}$ and cyclophanes. ${ }^{13}$ Despite the numerous macrocyclic structures obtained by

\footnotetext{
* Corresponding author: nbogdan@chem.ubbcluj.ro

${ }^{* *}$ Supplementary information on http: //web.icf.ro/rrch or http: //revroum.lew.ro
} 
CuAAC, its use in this field is somehow limited by the high dilution conditions imposed to favor macrocyclization over the oligomerization products which results in degradation of the catalyst before the reaction completion. ${ }^{14}$ Thus, to circumvent these issues, several catalytic systems were reported over the years displaying a moderate efficiency such as the use of copper tube flow reactors, ${ }^{15}$ immobilization of the catalyst on a solid support $^{16}$ or use of a variety of copper sources in presence of various heteroaryl ligands. ${ }^{14}$

In this context, we set to study the use of the copper(I)- $N$-heterocyclic carbene complex $\mathbf{I}^{17}$ (Figure 1) as single catalyst for the efficient synthesis of an unsymmetrical macrocycle through $\mathrm{CuAAC}$ click reaction without addition of any supplementary co-ligand.

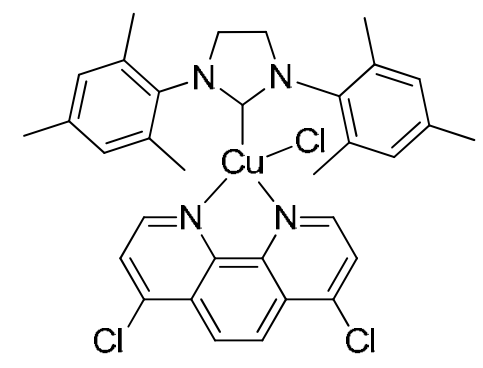

I

Fig. 1 - Structure of the copper(I)- $N$-heterocyclic carbene complex I used as catalyst in CuAAC reaction.

\section{RESULTS AND DISCUSSION}

\section{Synthesis of the macrocycle precursor}

The macrocycle presented in this work was designed to be obtained by intramolecular CuAAC reaction. Therefore we needed to prepare first a precursor that contained azide and ethynyl reactive groups in the same molecule. To do so, we synthesized two key intermediates: compound $\mathbf{4}$ containing the azide reactive group and compound 7 bearing the ethynyl functionality (Scheme 1).

Compound 4 was obtained in four steps: first the triethylene glycol was unsymmetrically chlorinated and the chlorine atom in $\mathbf{1}$ was further substituted with azide group. ${ }^{18}$ Activation of the hydroxyl group for the nucleophilic substitution by tosylation yielded compound $\mathbf{3}$ that was subsequently reacted with excess of hydroquinone to afford the target azido-decorated intermediate 4.

In order to synthesize the ethynyl functionalized derivative 7 , the tetraethylene glycol was first ditosylated ${ }^{19}$ and then reacted with a substoichiometric amount of monopropargyl hydroquinone 5 that was obtained by treatment of propargyl bromide with hydroquinone in large excess.

Next, the reaction between compounds $\mathbf{4}$ and 7 in presence of potassium carbonate yielded podand 8 in good yield ( $72 \%)$.

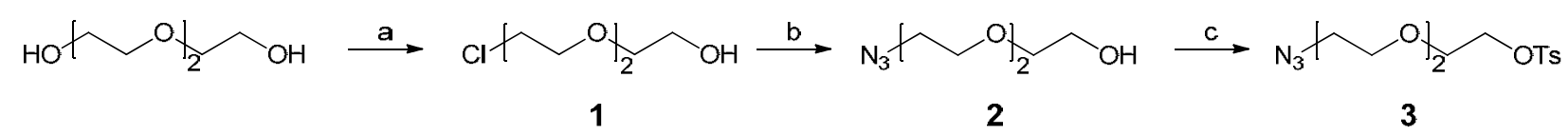

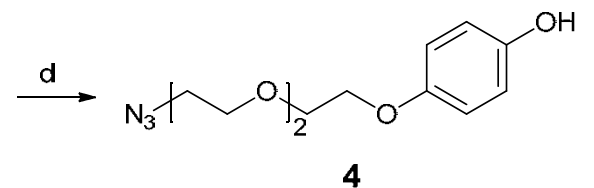

4

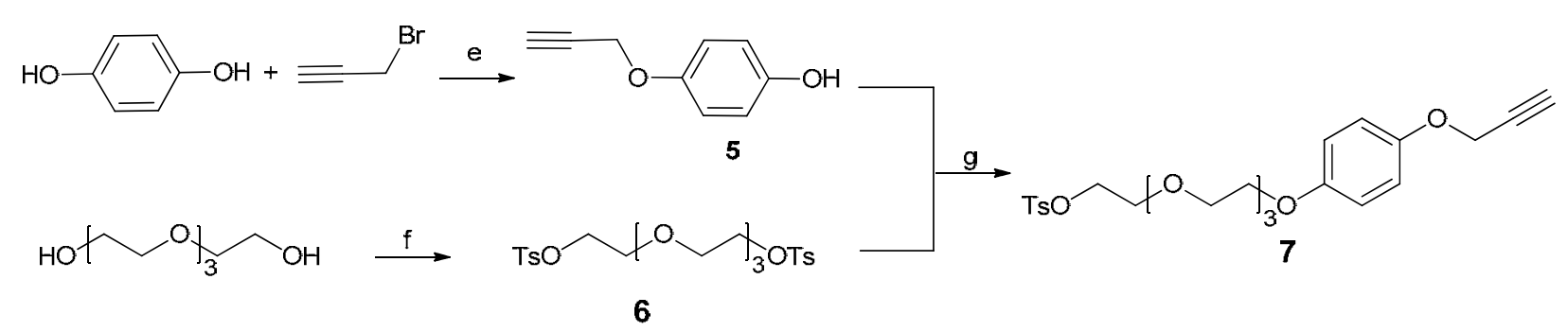<smiles>C#CCOc1ccc(OCCOCCOc2ccc(OCCOCCN)cc2)cc1</smiles>

Scheme 1 - Synthesis of the macrocycle precursor 8. Reagents and conditions: (a) $\mathrm{SOCl}_{2}, \mathrm{Py}, \mathrm{CHCl}_{3}, \mathrm{reflux}_{1}, 15 \%$; (b) $\mathrm{NaN}_{3}, \mathrm{NH}_{4} \mathrm{Cl}$, water, reflux, $92 \%$, (c) tosyl chloride, $\mathrm{KOH}, \mathrm{DCM}, 0^{\circ} \mathrm{C}, 85 \%$; (d) hydroquinone, $\mathrm{K}_{2} \mathrm{CO}_{3}$, acetonitrile, $15 \%$; (e) $\mathrm{K}_{2} \mathrm{CO}_{3}$, acetonitrile, reflux, $78 \%$; (f) tosyl chloride, $\mathrm{KOH}, \mathrm{DCM}, 0^{\circ} \mathrm{C}, 88 \%$; (g) $\mathrm{K}_{2} \mathrm{CO}_{3}$, acetone, reflux, 52\%; (h) $\mathrm{K}_{2} \mathrm{CO}_{3}$, acetonitrile, reflux, $72 \%$. 


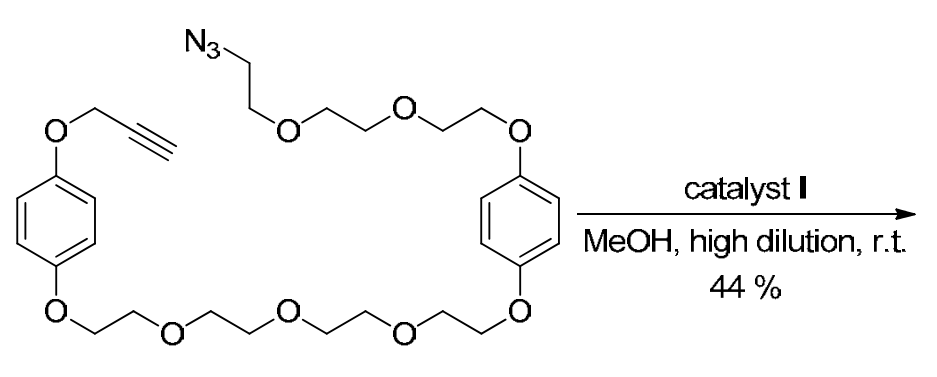

8

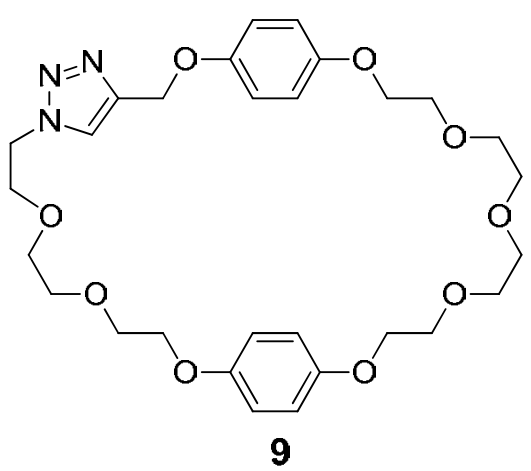

Scheme 2 - High-dilution, CuAAC synthesis of macrocycle 9 using copper(I)- $N$-heterocyclic carbene complex I as catalyst.

\section{Synthesis of the macrocycle 9}

With the precursor 8 in our hands we moved to the macrocyclization reaction by CuAAC (Scheme 2). Use of copper(I)- $N$-heterocyclic carbene complex $\mathbf{I}$ as catalyst allowed the macrocyclization reaction to conveniently occur. Thus, the catalyst was dissolved in methanol and the high dilution required to favour the formation of the macrocycle over the oligomerization products was ensured by slow addition ( 8 hours) of the solution of 8 in DCM/ methanol $(1 / 3 \mathrm{v} / \mathrm{v})$. In addition, the catalyst was airstable. ${ }^{17}$ Macrocycle 9 was obtained in $44 \%$ yield after purification by column chromatography.

Formation of macrocycle 9 was confirmed by NMR and HRMS spectra (see Supporting Information).

\section{Complexation studies}

The complexation ability of the macrocycle 9 towards alkali metal-ions was further investigated. Compound 9 was able to form complexes with all tested metal-ions as inferred from ESI(+)-HRMS experiments. The ESI(+)-HRMS spectra of the complexes obtained by treatment of macrocycle 9 $1.5 \times 10^{-3} \mathrm{M}$ in acetonitrile as solvent with 3 equivalents of $\mathrm{LiBF}_{4}, \mathrm{NaBF}_{4}, \mathrm{KBF}_{4}, \mathrm{RbBF}_{4}$ or $\mathrm{CsF}$ showed peaks corresponding to $\mathbf{9} \cdot \mathbf{M}^{+}$adducts at $m / z=580.2886 \quad\left[\mathrm{M}+\mathrm{Li}^{+}\right], \quad 596.2623 \quad\left[\mathrm{M}+\mathrm{Na}^{+}\right]$, $612.2365\left[\mathrm{M}+\mathrm{K}^{+}\right], \quad 658.1851, \quad\left[\mathrm{M}+\mathrm{Rb}^{+}\right]$and $706.1785\left[\mathrm{M}+\mathrm{Cs}^{+}\right]$respectively, as base peaks in all cases (see Supporting Informations Figure S18 to S22). Macrocycle 9 showed low affinity for ammonium ions. The ESI(+)-HRMS of the solution obtained by treatment of macrocycle 9 $1.5 \times 10^{-3} \mathrm{M}$ in acetonitrile with 3 equivalents of $\mathrm{NH}_{4} \mathrm{BF}_{4}$ displayed the protonated molecular ion of 9 at $m / z=574.2805$ as base peak and the peak corresponding to $\mathbf{9} \cdot \mathbf{N H}_{\mathbf{4}}{ }^{+}$adduct at $\mathrm{m} / \mathrm{z}=591.3066$ $\left[\mathrm{M}+\mathrm{NH}_{4}^{+}\right]$in about $4 \%$ intensity (see Supporting Information, Figure S23).

In order to investigate the selectivity of compound 9 for a particular metal-ion, competition complexation experiments were performed. Thus, 9 (1 equivalent) $1.5 \times 10^{-3} \mathrm{M}$ in acetonitrile was treated with a mixture containing equimolar amounts of $\mathrm{LiBF}_{4}, \mathrm{NaBF}_{4}, \mathrm{KBF}_{4}, \mathrm{RbBF}_{4}$ and $\mathrm{CsF}$ (1 equivalent each salt). The relative intensity of the peaks corresponding to $\left[\mathbf{9} \cdot \mathrm{Li}^{+}\right],\left[\mathbf{9} \cdot \mathrm{Na}^{+}\right],\left[\mathbf{9} \cdot \mathrm{K}^{+}\right]$, $\left[\mathbf{9} \cdot \mathrm{Rb}^{+}\right]$and $\left[\mathbf{9} \cdot \mathrm{Cs}^{+}\right]$adducts, determined from ESI(+)-HRMS spectrum (Figure 2) were $0 \%$, $100 \%, 92 \%, 73 \%$ and $95 \%$ respectively. The results were in agreement with a non-selective recognition behaviour toward the complexation of alkaline metal ions.

\section{EXPERIMENTAL}

General experimental data. All commercially available reagents were used without further purification. Thin layer chromatography (TLC) was performed on silica gel 60 coated aluminium $\mathrm{F}_{254}$ plates and visualised by $\mathrm{UV}$ irradiation at 254 $\mathrm{nm}$ or by staining with potassium permanganate solution. Preparative column chromatography was carried out using silica gel $60(0.040-0.063 \mathrm{~mm})$ from Merck. The NMR spectra were recorded on a Bruker Avance $400 \mathrm{MHz}$ or Bruker Avance $600 \mathrm{MHz}$ spectrometers. Chemical shifts $(\delta)$ are reported in parts per million (ppm) using residual solvent peak as internal reference. High resolution mass spectra were recorded on a Thermo Scientific (LTQ XL, Orbitrap) spectrometer, in positive ion mode, using Electrospray or APCI techniques.

\section{2-(2-(2-Chloroethoxy)ethoxy)ethanol (1)}

To a solution of triethylene glycol $(33.5 \mathrm{~mL}, 37.5 \mathrm{~g}, 250$ mmol, 1 eq.) in chloroform $(50 \mathrm{~mL})$, pyridine $(20.2 \mathrm{~mL}$, $19.75 \mathrm{~g}, 250 \mathrm{mmol}, 1 \mathrm{eq}$.) and $\mathrm{SOCl}_{2}(18.2 \mathrm{~mL}, 29.75 \mathrm{~g}, 250$ mmol, 1 eq.) were added and the reaction mixture was stirred under reflux for $24 \mathrm{~h}$. After cooling at room temperature DCM $(50 \mathrm{~mL})$ was added and the reaction mixture was washed with water $(40 \mathrm{~mL})$. Next, the aqueous phase was extracted with DCM $(2 \times 25 \mathrm{~mL})$. The combined organic phases were dried 
over anhydrous $\mathrm{MgSO}_{4}$ and the solvent was evaporated under vacuum. The crude of reaction contained compound $\mathbf{1}$ in a mixture with unreacted triethyleneglycol and the corresponding dichloro- derivative. Vacuum distillation (0.160.20 mbar) yielded three fractions, first fraction collected between $92-94^{\circ} \mathrm{C}$, the second fraction $99-106^{\circ} \mathrm{C}$ and a third fraction at over $109^{\circ} \mathrm{C}$. Third fraction contained the highest amount of $\mathbf{1}$. Further purification by column chromatography on silica gel, using a gradient of ethyl acetate : petroleum spirit, $40-60{ }^{\circ} \mathrm{C} 1: 2$ to $1: 0 \mathrm{v} / \mathrm{v}\left(\mathrm{R}_{\mathrm{f}}: 0.34\right)$ as eluent resulted in pure $5.96 \mathrm{~g}$ of $\mathbf{1}\left(15 \%\right.$ yield) as colourless liquid. ${ }^{\mathbf{1}} \mathbf{H}$ NMR (400 MHz, $\left.\mathrm{CDCl}_{3}\right), \delta_{\mathrm{H}}(\mathrm{ppm}): 3.60-3.66$ (overlapped peaks, $\left.4 \mathrm{H}, \mathrm{CH}_{2}\right), 3.69\left(\mathrm{~s}, 4 \mathrm{H}, \mathrm{CH}_{2}\right), 3.72-3.78$ (overlapped peaks, $\left.4 \mathrm{H}, \mathrm{CH}_{2}\right) .{ }^{13} \mathbf{C}$ APT NMR $\left(100 \mathrm{MHz}, \mathrm{CDCl}_{3}\right), \delta_{\mathrm{C}}(\mathrm{ppm}): 42.8$, $61.9,70.5,70.8,71.5,72.6$.

\section{2-(2-(2-Azidoethoxy)ethoxy)ethanol (2)}

To a solution of 1 ( $3 \mathrm{~g}, 17.8 \mathrm{mmol}, 1 \mathrm{eq}$.) in water (30 mL), $\mathrm{NaN}_{3}\left(11.57 \mathrm{~g}, 178 \mathrm{mmol}, 10\right.$ eq.) and $\mathrm{NH}_{4} \mathrm{Cl}(11.2$ g, $213.6 \mathrm{mmol}, 12$ eq.) were added. The resulted solution was stirred at $90{ }^{\circ} \mathrm{C}$ for $24 \mathrm{~h}$. After cooling at room temperature and filtration of the precipitated inorganic salts, the aqueous phase was extracted with ethyl acetate $(7 \times 40 \mathrm{~mL})$. The combined organic phases were dried over anhydrous $\mathrm{MgSO}_{4}$ and the solvent was evaporated under vacuum. We obtained $2.86 \mathrm{~g}$ (92\% yield) as a yellow liquid that was used in the next step without further purification. TLC (silica gel, ethyl acetate/ petroleum spirit, $\left.40-60{ }^{\circ} \mathrm{C}, 2: 3 \mathrm{v} / \mathrm{v}, \mathrm{R}_{\mathrm{f}}=0.34\right) .{ }^{1} \mathbf{H}$ NMR $\left(600 \mathrm{MHz}, \mathrm{CDCl}_{3}\right), \delta_{\mathrm{H}}(\mathrm{ppm}): 3.40\left(\mathrm{t},{ }^{3} J=5.0 \mathrm{~Hz}, 2 \mathrm{H}, \mathrm{CH}_{2}\right)$, $3.62\left(\mathrm{t},{ }^{3} \mathrm{~J}=4.4 \mathrm{~Hz}, 2 \mathrm{H}, \mathrm{CH}_{2}\right), 3.66-3.71$ (overlapped peaks, $\left.6 \mathrm{H}, \mathrm{CH}_{2}\right), 3.75\left(\mathrm{t},{ }^{3} \mathrm{~J}=4.4 \mathrm{~Hz}, 2 \mathrm{H}, \mathrm{CH}_{2}\right) .{ }^{13} \mathbf{C}$ APT NMR $(150$ $\left.\mathrm{MHz}, \mathrm{CDCl}_{3}\right), \delta_{\mathrm{C}}(\mathrm{ppm}): 50.8,61.9,70.2,70.6,70.8,72.6$.

\section{2-(2-(2-Azidoethoxy)ethoxy)ethyl 4-methylbenzenesulfonate (3)}

Compound 2 (2.62 g, $14.95 \mathrm{mmol}$, 1 eq.) and tosyl chloride $(2.85 \mathrm{~g}, 14.95 \mathrm{mmol}, 1$ eq.) were dissolved in DCM $(20 \mathrm{~mL})$. The solution was cooled on an ice bath and $\mathrm{KOH}$ ( $3.35 \mathrm{~g}, 59.8 \mathrm{mmol}, 4$ eq.) was added in small portions. The reaction mixture was stirred for $90 \mathrm{~min}$ at $0{ }^{\circ} \mathrm{C}$ and at room temperature for additional $20 \mathrm{~h}$. Next, water $(50 \mathrm{~mL})$ and DCM $(20 \mathrm{~mL})$ were added and the organic and aqueous phases were separated. The aqueous phase was extracted with DCM $(4 \times 20 \mathrm{~mL})$. The combined organic phases were washed with brine $(20 \mathrm{~mL})$ and dried over $\mathrm{MgSO}_{4}$. After evaporation of the solvent $4.17 \mathrm{~g}$ compound 3 (yellow liquid, $85 \%$ yield) were obtained. TLC ( (silica gel, ethyl acetate : petroleum spirit, $\left.40-60{ }^{\circ} \mathrm{C}, 1: 1, \mathrm{v} / \mathrm{v}, \mathrm{R}_{\mathrm{f}}=0.54\right) .{ }^{1} \mathrm{H}$ NMR $(600 \mathrm{MHz}$, $\left.\mathrm{CDCl}_{3}\right), \delta_{\mathrm{H}}(\mathrm{ppm}): 2.45\left(\mathrm{~s}, 3 \mathrm{H}, \mathrm{CH}_{3}\right), 3.37\left(\mathrm{t},{ }^{3} \mathrm{~J}=5.0 \mathrm{~Hz}, 2 \mathrm{H}\right.$, $\left.\mathrm{CH}_{2}\right), 3.60\left(\mathrm{~s}, 4 \mathrm{H}, \mathrm{CH}_{2}\right), 3.64\left(\mathrm{t},{ }^{3} J=5.0 \mathrm{~Hz}, 2 \mathrm{H}, \mathrm{CH}_{2}\right), 3.70$ $\left(\mathrm{t},{ }^{3} \mathrm{~J}=4.8 \mathrm{~Hz}, 2 \mathrm{H}, \mathrm{CH}_{2}\right), 4.16\left(\mathrm{t},{ }^{3} J=4.8 \mathrm{~Hz}, 2 \mathrm{H}, \mathrm{CH}_{2}\right), 7.34$ $\left(\mathrm{d},{ }^{3} \mathrm{~J}=8.2 \mathrm{~Hz}, 2 \mathrm{H}, \mathrm{CH}_{\mathrm{Ar}}\right), 7.80\left(\mathrm{~d},{ }^{3} J=8.2 \mathrm{~Hz}, 2 \mathrm{H}, \mathrm{CH}_{\mathrm{Ar}}\right) .{ }^{13} \mathbf{C}$ APT NMR $\left(150 \mathrm{MHz}, \mathrm{CDCl}_{3}\right), \delta_{\mathrm{C}}(\mathrm{ppm}): 50.8,60.5,69.0$, $69.4,70.2,70.8,71.0,128.1,130.0,133.2,145.0$.

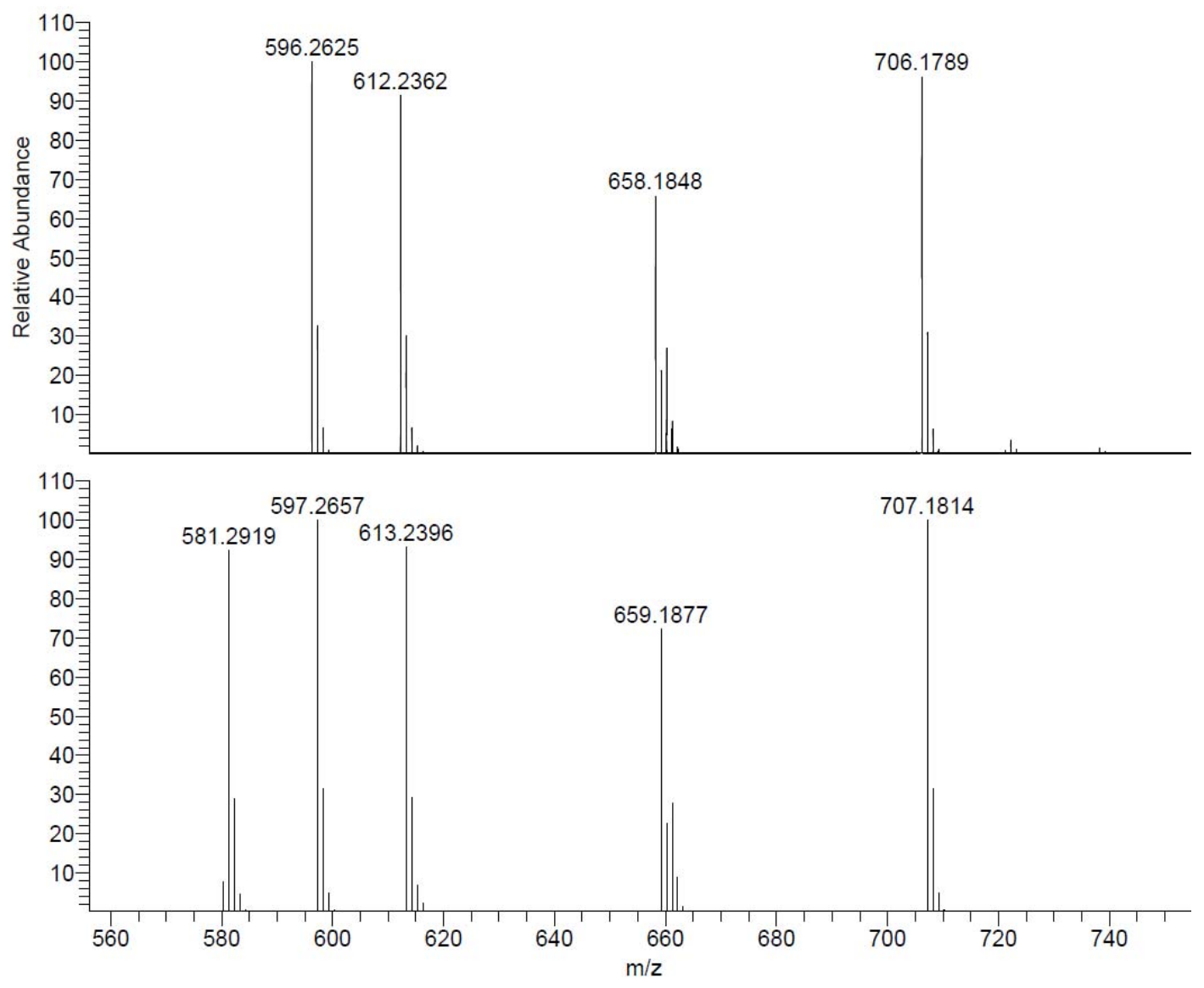

Fig. 2 - ESI(+)-HRMS spectra of $91.5 \mathrm{mM}$ in acetonitrile, in presence of equimolar amounts of $\mathrm{LiBF}_{4}, \mathrm{NaBF}_{4}$, $\mathrm{KBF}_{4}, \mathrm{RbBF}_{4}$ and $\mathrm{CsF}$ (top). Comparison of the experimental spectra (top) and simulated isotopic patterns of $\left[\mathbf{9} \mathrm{Li}^{+}\right],\left[\mathbf{9} \cdot \mathrm{Na}^{+}\right],\left[\mathbf{9} \cdot \mathrm{K}^{+}\right],\left[\mathbf{9} \mathrm{Rb}^{+}\right]$and $\left[\mathbf{9} \cdot \mathrm{Cs}^{+}\right]$adducts (bottom). 


\section{4-(2-(2-(2-azidoethoxy)ethoxy)ethoxy)phenol (4)}

A solution of compound $\mathbf{3}$ (3.796 g, $11.54 \mathrm{mmol}, 1$ eq.) in acetonitrile $(150 \mathrm{~mL})$ was flushed with argon, then hydroquinone (6.347 g, $57.7 \mathrm{mmol}, 5$ eq.) and $\mathrm{K}_{2} \mathrm{CO}_{3}(15.92 \mathrm{~g}$, $115.4 \mathrm{mmol}$ ) were added. The reaction mixture was stirred at reflux, under argon atmosphere, for $24 \mathrm{~h}$. After evaporation of the solvent, water $(150 \mathrm{~mL})$ was added and the mixture was extracted with ethyl acetate $(5 \times 50 \mathrm{~mL})$. The combined organic phases were washed with brine $(100 \mathrm{~mL})$, dried over anhydrous $\mathrm{MgSO}_{4}$ and the solvent evaporated under vacuum. The crude of reaction was purified by column chromatography using silica gel as stationary phase and a gradient of ethyl acetate: petroleum spirit, $40-60{ }^{\circ} \mathrm{C} 2: 3$ to $1: 1 \mathrm{v} / \mathrm{v}$ as eluent. Compound $40.46 \mathrm{~g}$ ( $15 \%$ yield) was obtained as a brown oil. TLC (silica gel, ethyl acetate : petroleum spirit, $40-60{ }^{\circ} \mathrm{C} 1: 2$, $\left.\mathrm{R}_{\mathrm{f}}=0.22\right){ }^{1} \mathbf{H}$ NMR $\left(600 \mathrm{MHz}, \mathrm{CDCl}_{3}\right), \delta_{\mathrm{H}}(\mathrm{ppm}): 3.39(\mathrm{t}$, ${ }^{3} J=5.0 \mathrm{~Hz}, 2 \mathrm{H}, \mathrm{CH}_{2}$ ), 3.66-3.71 (overlapped signals, $4 \mathrm{H}$, $\left.\mathrm{CH}_{2}\right), 3.72-3.75\left(\mathrm{~m}, 2 \mathrm{H}, \mathrm{CH}_{2}\right), 3.84\left(\mathrm{t},{ }^{3} \mathrm{~J}=4.8 \mathrm{~Hz}, 2 \mathrm{H}, \mathrm{CH}_{2}\right)$, $4.07\left(\mathrm{t},{ }^{3} \mathrm{~J}=4.9 \mathrm{~Hz}, 2 \mathrm{H}, \mathrm{CH}_{2}\right), 4.51$ (br.s, $\left.1 \mathrm{H}, \mathrm{OH}\right), 6.75\left(\mathrm{~d},{ }^{3} J\right.$ $\left.=9.0 \mathrm{~Hz}, 2 \mathrm{H}, \mathrm{CH}_{\mathrm{ar}}\right), 6.80\left(\mathrm{~d},{ }^{3} \mathrm{~J}=9.0 \mathrm{~Hz}, 2 \mathrm{H}, \mathrm{CH}_{\mathrm{ar}}\right) .{ }^{13} \mathbf{C}$ APT NMR $\left(150 \mathrm{MHz}, \mathrm{CDCl}_{3}\right), \delta_{\mathrm{C}}(\mathrm{ppm}): 50.9,68.3,70.1,70.2$, 70.9, 71.0, 116.0, 116.2, 149.8, 153.2. ESI(+)-HRMS $(\mathrm{m} / \mathrm{z})$ : Calculated for $\mathrm{C}_{12} \mathrm{H}_{17} \mathrm{~N}_{3} \mathrm{O}_{4}: 290.1111$, found 290.1134 $\left[\mathrm{M}+\mathrm{Na}^{+}\right]$.

\section{4-(Prop-2-yn-1-yloxy)phenol (5)}

In a $250 \mathrm{~mL}$ flask, propargyl bromide $(1.75 \mathrm{~g}, 14.7 \mathrm{mmol}$ 1 eq.), hydroquinone ( $8 \mathrm{~g}, 72.7 \mathrm{mmol}, 5$ eq.) and $\mathrm{K}_{2} \mathrm{CO}_{3}$ $(40.57 \mathrm{~g}, 294 \mathrm{mmol}, 20 \mathrm{eq})$ in acetonitrile $(200 \mathrm{~mL})$ were added. The reaction mixture was stirred under reflux for $24 \mathrm{~h}$. After completion of the reaction, the solid was removed by filtration and the solvent was evaporated in vacuum. The crude of reaction was purified by column chromatography using silica gel as stationary phase and a gradient of ethyl acetate petroleum spirit, $40-60{ }^{\circ} \mathrm{C} 1: 4$ to $1: 2$ as eluent. We obtained $1.69 \mathrm{~g}$ of 5 (78 \% yield). TLC (silica gel, ethyl acetate : petroleum spirit, $\left.40-60{ }^{\circ} \mathrm{C} 1: 3, \mathrm{R}_{\mathrm{f}}=0.34\right) .{ }^{1} \mathbf{H}$ NMR $\left(600 \mathrm{MHz}, \mathrm{CD}_{3} \mathrm{OD}\right), \delta_{\mathrm{H}}(\mathrm{ppm}): 2.89\left(\mathrm{t},{ }^{4} J=2.3 \mathrm{~Hz}, 1 \mathrm{H}, \mathrm{CH}\right)$, $4.61\left(\mathrm{~d},{ }^{4} J=2.3 \mathrm{~Hz}, 2 \mathrm{H}, \mathrm{CH}_{2}\right), 6.70\left(\mathrm{~d},{ }^{3} J=8.9 \mathrm{~Hz}, 2 \mathrm{H}\right.$, $\left.\mathrm{CH}_{\mathrm{Ar}}\right), 6.82\left(\mathrm{~d},{ }^{4} \mathrm{~J}=8.9 \mathrm{~Hz}, 2 \mathrm{H}, \mathrm{CH}_{\mathrm{Ar}}\right) \cdot{ }^{13} \mathbf{C}$ APT NMR $(150$ $\left.\mathrm{MHz}, \mathrm{CD}_{3} \mathrm{OD}\right), \delta_{\mathrm{C}}(\mathrm{ppm}): 56.8,75.5,79.0,116.2,116.5$, 150.4, 151.9.

\section{((Oxy-bis(ethane-2,1-diyl))-bis(oxy))-bis(ethane-2,1-diyl)- bis(4-methylbenzenesulfonate) (6)}

Tetraethylene glycol (29.13 g, $150 \mathrm{mmol}, 1$ eq.) in $\mathrm{CH}_{2} \mathrm{Cl}_{2}$ $(150 \mathrm{~mL})$ was treated with tosyl chloride $(57.2 \mathrm{~g}, 300 \mathrm{mmol}, 2$ eq.). The mixture was cooled on an ice bath and $\mathrm{KOH}(67.2 \mathrm{~g}$, 1.2 mol, 8 eq.) was added in small portions. The reaction mixture was stirred at $0{ }^{\circ} \mathrm{C}$ for $3 \mathrm{~h}$ and at room temperature for additional $20 \mathrm{~h}$. After this time, water $(300 \mathrm{~mL})$ and DCM $(150$ $\mathrm{mL}$ ) were added and the organic phase was separated and washed with water $(100 \mathrm{~mL})$. The combined aqueous phases were extracted with DCM $(1 \times 150 \mathrm{~mL}$ and $1 \times 100 \mathrm{~mL})$. The combined organic phases were washed with water $(100 \mathrm{~mL})$, dried over $\mathrm{MgSO}_{4}$ and the solvent was evaporated in vacuum. We obtained $66.18 \mathrm{~g}$ of 6 ( $88 \%$ yield) as a colourless liquid. TLC (silica gel, ethyl acetate: petroleum spirit, $40-60{ }^{\circ} \mathrm{C} 1: 1$, $\left.\mathrm{R}_{\mathrm{f}}=0.26\right) .{ }^{1} \mathrm{H}$ NMR $\left(400 \mathrm{MHz}, \mathrm{CDCl}_{3}\right), \delta_{\mathrm{H}}(\mathrm{ppm}): 2.44$ (s, $6 \mathrm{H}$, $\mathrm{CH}_{3}$ ), 3.53-3.59 (overlapped signals, $8 \mathrm{H}, \mathrm{CH}_{2}$ ), 3.68 (t, $\left.{ }^{3} J=4.8 \mathrm{~Hz}, 4 \mathrm{H}, \mathrm{CH}_{2}\right), 4.15\left(\mathrm{t},{ }^{3} \mathrm{~J}=4.8 \mathrm{~Hz}, 4 \mathrm{H}, \mathrm{CH}_{2}\right), 7.34(\mathrm{~d}$, $\left.{ }^{3} J=8.2 \mathrm{~Hz}, 4 \mathrm{H}, \mathrm{CH}_{\mathrm{Ar}}\right), 7.79\left(\mathrm{~d},{ }^{3} J=8.2 \mathrm{~Hz}, 4 \mathrm{H}, \mathrm{CH}_{\mathrm{Ar}}\right) .{ }^{13} \mathbf{C}$ APT NMR (150 MHz, $\left.\mathrm{CDCl}_{3}\right), \delta_{\mathrm{C}}$ (ppm): 21.8, 68.8, 69.4, 70.7, 70.9, 128.1, 130.0, 133.2, 145.0.
2-(2-(2-(2-(4-(Prop-2-yn-1-yloxy)phenoxy)ethoxy)ethoxy) ethoxy)ethyl 4-methylbenzenesulfonate (7) Compound $5(0.8 \mathrm{~g}$, $5.4 \mathrm{mmol}, 1$ eq.), compound 6 (10.84 g, $21.6 \mathrm{mmol}, 4$ eq.) and $\mathrm{K}_{2} \mathrm{CO}_{3}(7.45 \mathrm{~g}, 54 \mathrm{mmol}, 10$ eq.) were dissolved in acetone $(125 \mathrm{~mL})$. The reaction mixture was stirred under reflux for 48h. After filtration, the solvent was evaporated and the residue was purified by column chromatography on silica gel using a gradient of ethyl acetate: petroleum spirit, $40-60{ }^{\circ} \mathrm{C}$ $1: 2$ to $1: 1 \mathrm{v} / \mathrm{v}$ ) to give $1.35 \mathrm{mg}$ compound 7 (52\% yield) as a colourless oil. TLC (silica gel, ethyl acetate : petroleum spirit, 40-60 $\left.{ }^{\circ} \mathrm{C} 2: 1, \mathrm{R}_{\mathrm{f}}=0.46\right) .{ }^{1} \mathrm{H}$ NMR $\left(600 \mathrm{MHz}, \mathrm{CDCl}_{3}\right), \delta_{\mathrm{H}}$ (ppm): 2.44 (s, $\left.3 \mathrm{H}, \mathrm{CH}_{3}\right), 2.50\left(\mathrm{t},{ }^{4} \mathrm{~J}=2.4 \mathrm{~Hz}, 1 \mathrm{H}, \mathrm{CH}\right), 3.56-$ 3.61 (overlapped signals, $\left.4 \mathrm{H}, \mathrm{CH}_{2}\right), 3.63-3.65\left(\mathrm{~m}, 2 \mathrm{H}, \mathrm{CH}_{2}\right)$, 3.67-3.73 (overlapped signals, $\left.4 \mathrm{H}, \mathrm{CH}_{2}\right), 3.81-3.84(\mathrm{~m}, 2 \mathrm{H}$, $\left.\mathrm{CH}_{2}\right), 4.06-4.09\left(\mathrm{~m}, 2 \mathrm{H}, \mathrm{CH}_{2}\right), 4.13-4.16\left(\mathrm{~m}, 2 \mathrm{H}, \mathrm{CH}_{2}\right), 4.64$ $\left(\mathrm{d},{ }^{4} \mathrm{~J}=2.3 \mathrm{~Hz}, 2 \mathrm{H}, \mathrm{CH}_{2}\right), 6.85\left(\mathrm{~d},{ }^{3} \mathrm{~J}=9.1 \mathrm{~Hz}, 2 \mathrm{H}, \mathrm{CH}_{\mathrm{Ar}}\right)$, $6.90\left(\mathrm{~d},{ }^{3} J=9.1 \mathrm{~Hz}, 2 \mathrm{H}, \mathrm{CH}_{\mathrm{Ar}}\right), 7.33\left(\mathrm{~d},{ }^{3} J=8.1 \mathrm{~Hz}, 2 \mathrm{H}\right.$, $\left.\mathrm{CH}_{\mathrm{Ar}}\right), 7.79\left(\mathrm{~d},{ }^{3} \mathrm{~J}=8.3 \mathrm{~Hz}, 2 \mathrm{H}, \mathrm{CH}_{\mathrm{Ar}}\right) .{ }^{13} \mathbf{C}$ APT NMR $(150$ $\left.\mathrm{MHz}, \mathrm{CDCl}_{3}\right), \delta_{\mathrm{C}}(\mathrm{ppm}): 21.7,56.6,68.1,68.7,69.3,69.9$, 70.6, 70.7, 70.8 (2C), 75.4, 79.0, 115.6, 116.2, 128.0, 129.9, 133.1, 144.9, 151.9, 153.7. ESI $(+)-H R M S(m / z)$ : Calculated for $\mathrm{C}_{24} \mathrm{H}_{30} \mathrm{O}_{8} \mathrm{~S}: 501.1554$, found $501.1590\left[\mathrm{M}+\mathrm{Na}^{+}\right]$.

1-(2-(2-(2-azidoethoxy)ethoxy)ethoxy)-4-(2-(2-(2-(2-(4(prop-2-yn-1-yloxy)phenoxy) ethoxy)ethoxy) ethoxy)ethoxy) benzene (8)

Compound 4 (151 mg, $0.565 \mathrm{mmol}, 1.1$ eq. $)$ and compound 7 ( $247 \mathrm{mg}, 0.514 \mathrm{mmol}, 1 \mathrm{eq}$.) were dissolved in acetonitrile $(15 \mathrm{~mL})$ and $\mathrm{Cs}_{2} \mathrm{CO}_{3}(838 \mathrm{mg}, 2.57 \mathrm{mmol}, 5$ eq.) was added. The mixture was flashed with argon and stirred for $24 \mathrm{~h}$ under reflux. After evaporation of the solvent, an aqueous solution of $\mathrm{NaOH} 10 \%(20 \mathrm{~mL})$ was added and the mixture was extracted with ethyl acetate $(2 \times 50 \mathrm{~mL}$ and $4 \times 25 \mathrm{~mL})$. The combined organic phases were washed with brine and dried over anhydrous $\mathrm{MgSO}_{4}$. The solvent was evaporated under vacuum and the crude of reaction was purified by column chromatography on silica gel using a mixture of ethyl acetate: petroleum spirit, $\mathrm{v} / \mathrm{v} \quad 40-60{ }^{\circ} \mathrm{C} 2: 1 \mathrm{v} / \mathrm{v}$ as eluent $\left(\mathrm{R}_{\mathrm{f}}=0.55\right)$. We obtained $0.232 \mathrm{~g}$ of $\mathbf{8}(72 \%$ yield $)$ as a yellowish oil. ${ }^{1} \mathbf{H}$ NMR $\left(600 \mathrm{MHz}, \mathrm{CDCl}_{3}\right), \delta_{\mathrm{H}}(\mathrm{ppm}): 2.50(\mathrm{t}$, $\left.{ }^{4} J=2.3 \mathrm{~Hz}, 1 \mathrm{H}, \mathrm{CH}\right), 3.39\left(\mathrm{t},{ }^{3} J=5.0 \mathrm{~Hz}, 2 \mathrm{H}, \mathrm{CH}_{2}\right), 3.66-3.70$ (overlapped signals, $8 \mathrm{H}, \mathrm{CH}_{2}$ ), 3.70-3.75 (overlapped signals, $6 \mathrm{H}, \mathrm{CH}_{2}$ ), 3.81-3.85 (overlapped signals, $6 \mathrm{H}, \mathrm{CH}_{2}$ ), 4.04-4.09 (overlapped signals, $\left.6 \mathrm{H}, \mathrm{CH}_{2}\right), 4.63\left(\mathrm{~d},{ }^{4} J=2.3 \mathrm{~Hz}, 2 \mathrm{H}, \mathrm{CH}_{2}\right)$, $6.83\left(\mathrm{~s}, 4 \mathrm{H}, \mathrm{CH}_{\mathrm{ar}}\right), 6.85\left(\mathrm{~d},{ }^{3} \mathrm{~J}=9.1 \mathrm{~Hz}, 2 \mathrm{H}, \mathrm{CH}_{\mathrm{Ar}}\right), 6.90(\mathrm{~d}$, $\left.{ }^{3} J=9.1 \mathrm{~Hz}, 2 \mathrm{H}, \mathrm{CH}_{\mathrm{Ar}}\right) .{ }^{13} \mathbf{C}$ APT NMR $\left(150 \mathrm{MHz}, \mathrm{CDCl}_{3}\right)$, $\delta_{\mathrm{C}}(\mathrm{ppm}): 50.9,56.7,68.2(2 \mathrm{C}), 70.0(2 \mathrm{C}), 70.1,70.2,70.8$ (2C), 70.9, 71.0 (3C), 71.0, 75.4, 79.0, 115.7 (3C), 116.2, 152.0, 153.3 (2C), 153.9. ESI(+)-HRMS $(\mathrm{m} / \mathrm{z})$ : Calculated for $\mathrm{C}_{29} \mathrm{H}_{39} \mathrm{~N}_{3} \mathrm{O}_{9}$ : 596.2579, found 596.2621 [M+Na $\left.{ }^{+}\right]$.

\section{2,10,13,16,21,24,27,30,33-nonaoxa-5,6,7-triazatetracyclo [32.2.2.2 $2^{17,20} \cdot 1^{4,7}$ ]hentetraconta-1(36),4(41),5, \\ 17,19,34,37,39-octaene (9)}

In a two-neck flask, methanol $(100 \mathrm{~mL})$ was added and flushed with argon for $10 \mathrm{~min}$. Then, copper(I) $N$-heterocyclic carbene complex I (12 mg, $0.0183 \mathrm{~mol}, 7 \mathrm{~mol} \%$.) was added. A solution of $8(152 \mathrm{mg}, 0.265 \mathrm{mmol})$ dissolved in methanol/DCM ( $4 \mathrm{~mL}, 3 / 1 \mathrm{v} / \mathrm{v})$ was added over 8 hours using a push-syringe and the reaction mixture was stirred at room temperature, under argon, for 3 days. After evaporation of the solvent, water $(10 \mathrm{~mL})$ was added and the mixture was extracted with ethyl acetate $(1 \times 20 \mathrm{~mL}$ and $3 \times 10 \mathrm{~mL})$. The combined organic phases were dried over anhydrous $\mathrm{MgSO}_{4}$ 
and evaporated. After purification by column chromatography on silica gel using a mixture of ethyl acetate and methanol 9:1 $\mathrm{v} / \mathrm{v}$ as elution system $\left(\mathrm{R}_{\mathrm{f}}=0.49\right) 67 \mathrm{mg}$ of macrocycle $9(44 \%$ yield) were obtained as colourless oil . ${ }^{1} \mathbf{H}$ NMR $(600 \mathrm{MHz}$, $\left.\mathrm{CDCl}_{3}\right), \delta_{\mathrm{H}}(\mathrm{ppm}): 3.63-3.66\left(\mathrm{~m}, 2 \mathrm{H}, \mathrm{CH}_{2}\right), 3.66-3.77$ (overlapped peaks, $12 \mathrm{H}, \mathrm{CH}_{2}$ ), 3.86 (overlapped peaks, $6 \mathrm{H}$, $\mathrm{CH}_{2}$ ), 3.93-3.99 (overlapped peaks, $\left.4 \mathrm{H}, \mathrm{CH}_{2}\right), 4.03(t$, $\left.{ }^{3} J=4.8 \mathrm{~Hz}, 2 \mathrm{H}, \mathrm{CH}_{2}\right), 4.47\left(\mathrm{t},{ }^{3} J=4.8 \mathrm{~Hz}, 2 \mathrm{H}, \mathrm{CH}_{2}\right), 4.99$ (s, $\left.2 \mathrm{H}, \mathrm{CH}_{2}\right), 6.67\left(\mathrm{~s}, 4 \mathrm{H}, \mathrm{CH}_{\mathrm{Ar}}\right), 6.76\left(\mathrm{~d},{ }^{3} J=9.3 \mathrm{~Hz}, 2 \mathrm{H}, \mathrm{CH}_{\mathrm{Ar}}\right)$, $6.78\left(\mathrm{~d},{ }^{3} \mathrm{~J}=9.3 \mathrm{~Hz}, 2 \mathrm{H}, \mathrm{CH}_{\mathrm{Ar}}\right), \delta 7.97\left(\mathrm{~s}, 1 \mathrm{H}, \mathrm{CH}_{\text {triazole }}\right) .{ }^{13} \mathbf{C}$ APT NMR $\left(150 \mathrm{MHz}, \mathrm{CDCl}_{3}\right), \delta_{\mathrm{C}}(\mathrm{ppm}): 50.7,62.4,68.1$, $68.3,68.4,69.3,69.9,70.0$ (2C), 70.5, 70.7, 70.8, 70.9 (2C), 71.2, 115.5, 115.7, 115.8 (2C), 124.7, 143.9, 152.7, 153.0, 153.4, 153.6. $\quad$ ESI $(+)-H R M S \quad(m / z): \quad$ Calculated for $\mathrm{C}_{29} \mathrm{H}_{39} \mathrm{~N}_{3} \mathrm{O}_{9}: 574.2759$, found $574.2789\left[\mathrm{M}+\mathrm{H}^{+}\right]$.

\section{CONCLUSIONS}

In summary, we described synthesis and characterization of a new triazole-based unsymmetrical macrocycle, using a precusrsor that contained in the same molecule two reactive functional groups. The triazole ring was efficienly formed in the macrocyclization step by $\mathrm{CuAAC}$ reaction in presence of a copper(I)- $N$-heterocyclic carbene complex as catalyst. Complexation experiments showed that the synthesized macrocycle was able to recognize all the investigated alkali metal-ions, namely $\mathrm{Li}^{+}, \mathrm{Na}^{+}, \mathrm{K}^{+}, \mathrm{Rb}^{+}$and $\mathrm{Cs}^{+}$. However, the competition experiments were in agreement with a non-selective complexation behaviour. The high macrocyclisation yield and the ease of the procedure promts toward future design and synthesis of more selective and specific triazole-based macroxyclic receptors for metal ions.

Acknowledgments. Financial support by Roumanian National Authority for Scientific Research and Innovation, CNCS-UEFISCDI, project number PN-III-P4-ID-PCCF-20160088, is gratefully acknowledged. We thank Dr. Arnaud Gautier for providing us the catalyst used in CuAAC reaction. Teodor Cucuiet thanks to Babeş-Bolyai University for a one year scholarship.

Supporting Information Available. Full spectroscopic data for all new compounds. HR-MS spectra of the alkali-metal ions complexes.

\section{REFERENCES}

1. (a) E. Weber, F. Vögtle and A. K. Burrell, "Macrocycles", Springer-Verlag, 1992; (b) J.-M. Lehn, "Supramolecular Chemistry. Concepts and Perspectives," Wiley-VCH Verlag $\mathrm{GmbH}, 1995$.

2. M. Matache, E. Bogdan and N. D. Hădade, Chem. Eur. J., 2014, 20, 2106-2131.

3. D. Pasini, "Synthesis of Macrocycles and Click Chemistry" in "Click Reactions in Organic Synthesis" Wiley VCH Verlag GmbH, 2016, p. 287-307.

4. L. Xu, Y. Li and Y. Li, Asian J. Org. Chem., 2014, 3, 582602.

5. (a) P. L. Golas and K. Matyjaszewski, Chem. Soc. Rev., 2010, 39, 1338-1354; (b) K. Kacprzak, I. Skiera, M. Piasecka and Z. Paryzek, Chem. Rev., 2016, 116, 56895743; (c) L. Liang and D. Astruc, Coord. Chem. Rev., 2011, 255, 2933-2945.

6. J. Hou, X. Liu, J. Shen, G. Zhao and P. G. Wang, Expert Opin. Drug Discov., 2012, 7, 489-501.

7. B. E. Fletcher, M. J. G. Peach and N. H. Evans, Org. Biomol. Chem., 2017, 15, 2797-2803.

8. K. D. Hänni and D. A. Leigh, Chem. Soc. Rev., 2010, 39, 1240-1251.

9. (a) R. Peng, Y. Xu and Q. Cao, Chinese Chem. Lett., 2018, 29, 1465-1474; (b) D. Pasini, Molecules, 2013, 18, 95129530.

10. J. M. Holub and K. Kirshenbaum, Chem. Soc. Rev., 2010, 39, 1325-1337.

11. (a) A. Ajay, S. Sharma, M. P. Gupt, V. Bajpai, H. B. Kumar, M. P. Kaushik, R. Konwar, R. S. Ampapathi and R. P. Tripathi, Org. Lett., 2012, 14, 4306-4309; (b) V. L. Campo, I. M. Ivanova, I. Carvalho, C. D. Lopes, Z. A. Carneiro, G. Saalbach, S. Schenkman, J. Santana da Silva, S. A. Nepogodiev and R. A. Field, Tetrahedron, 2015, 71, 7344 7353; (c) A. A. Salman, M. Tabandeh, T. Heidelberg and R. S. D. Hussen, Carbohyd. Res., 2015, 406, 41-45.

12. (a) Y. S. L. Choo, P. G. Waddell and A. C. Benniston, Eur. J. Org. Chem., 2019, 2560-2565; (b) J. Romański and P. Jaworski, Phosphorus, Sulfur, Silicon Relat. Elem., 2017, 192, 231-234.

13. Y. Yu, Y. Li, S Chen, T. Liu, Z. Qin, H. Liu and Y. Li, Org. Lett., 2016, 18, 3394-3397.

14. G. Chouhan and K. James, Org. Lett., 2011, 13, 2754-2757.

15. A. R. Bogdan and K. James, Chem. Eur. J., 2010, 16, 14506-14512.

16. V. Haridas, K. Lal, Y. K. Sharma and S. Upreti, Org. Lett., 2008, 10, 1645-1647.

17. P. Bruyat, A. Gautier, L. Jean and P.-Y. Renard, J. Org. Chem., 2018, 83, 13515-13522.

18. N. D. Bogdan, M. Matache, V. M. Meier, C. Dobrotă, I. Dumitru, G. D. Roiban and D. P. Funeriu, Chem. Eur. J., 2010, 16, 2170-2180.

19. K. M. Bonger, R. J. B. H. N. Van den Berg, L. H. Heitman, A. P. IJzerman, J. Oosterom, C. M. Timmers, H. S. Overkleeft and G. A. van der Marel, Bioorg. Med. Chem., 2007, 15, 4841-4856. 